Planetary Systems in the Universe - Observation, Formation and Evolution

Proceedings IAU Symposium No. 202, (C)2004 IAU

Alan Penny, Pawel Artymowicz, Anne-Marie Lagrange, \& Sara Russell, eds.

\title{
Orbital Eccentricity Growth through Disc-Companion Tidal Interactions
}

\author{
Richard P. Nelson, John C.B. Papaloizou and Frédéric Masset \\ Astronomy Unit, School of Mathematical Sciences, Queen Mary, \\ University of London, Mile End Road, London E1 4NS, UK
}

\begin{abstract}
We present the results of 2-D non linear hydrodynamic simulations performed to examine the driving of orbital eccentricity through disccompanion tidal interaction. We examine the eccentricity evolution for companion masses, $m_{p}$, in the range $1 \leq m_{p} \leq 30 \mathrm{M}_{\text {Jupiter. }}$. The disc model we employ is similar to a minimum mass solar nebula model.

We find that there is a transition in behaviour, characterised by a rapid growth in eccentricity, for companion masses $m_{p} \gtrsim 20 \mathrm{M}_{\text {Jupiter }}$. Companions with masses below this limit show essentially no growth in eccentricity, for the disc model parameters that we examine. Our results suggest that if giant planets form from protostellar discs with properties similar to those studied in this paper, then the observed orbital eccentricities of the extrasolar planets are not a consequence of tidal interaction with their protostellar discs during formation.
\end{abstract}

\section{Introduction}

The recent discovery of extrasolar planets orbiting nearby solar-type stars (e.g. Marcy, Cochran, \& Mayor 2000) has stimulated renewed interest in the theory of planet formation. In particular, the major challenge now facing planet formation theories is to provide an explanation of the current data.

Working within the framework of the 'core instability' model of planet formation, the observation that there exists an upper limit to the masses of planets, $m_{p}$, such that $m_{p}<10 \mathrm{M}_{\text {Jupiter }}$ may be explained as a consequence of gap formation and the finite lifetimes of protostellar discs (Bryden et al. 1999; Kley 1999; Lubow, Seibert, \& Artymowicz 1999). In addition, the shorter period systems and 'hot Jupiters' may be explained as a consequence of inward migration caused by tidal interaction between a protostellar disc and an embedded protoplanet (Lin \& Papaloizou 1986; Nelson et al. 2000). However, the fact that many planets are on eccentric orbits has so far not been explained.

The work presented here explores the possibility that tidal interaction between a protostellar disc and an embedded companion may lead to the growth of orbital eccentricity. Previous studies of disc-companion interaction suggest that eccentricity may be driven by resonant wave excitation at the 1:3 outer eccentric Lindblad resonance (Artymowicz 1992; Lin \& Papaloizou 1993). However, a wide gap is required to remove coorbital material that would damp the eccentricity through coorbital Lindblad torques, so that the onset of eccentricity growth is expected to be a function of planetary mass and disc parameters. Here we present the results of numerical simulations designed to determine for which 


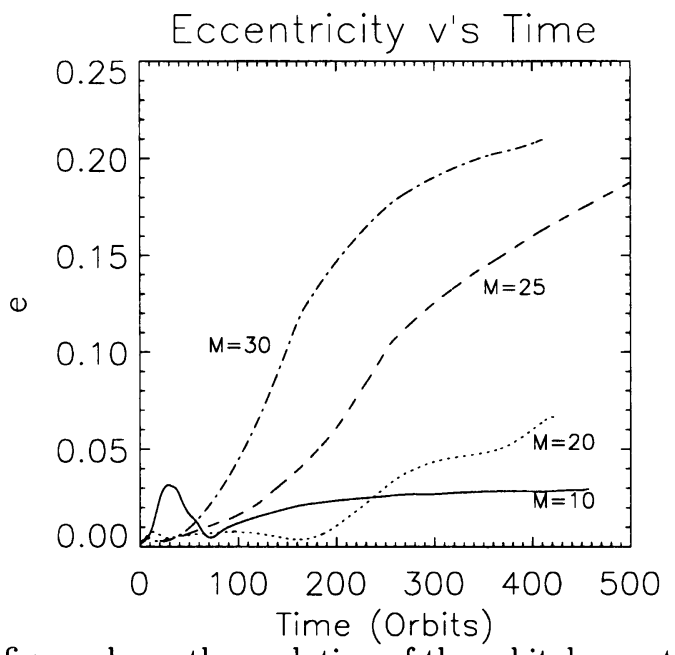

Figure 1. This figure shows the evolution of the orbital eccentricity. Time is measured in companion orbits. Each line is labelled with the corresponding companion mass, in units of a Jupiter mass.

companion mass, if any, eccentricity growth occurs due to interaction with a disc that is similar to a minimum mass solar nebula model.

\section{Numerical Calculations}

The simulations were performed using a modified version of the Eulerian gridbased code 'NIRVANA' (Ziegler \& Yorke 1997). The Keplerian disc aspect ratio was taken to be $H / r=0.05$, and the viscosity parameter $\alpha=6 \times 10^{-3}$. The disc surface density $\Sigma$ was taken to be constant, except at the outer edge where a taper was applied, and was normalised so that 2 Jupiter masses lay within the companion orbit radius at time $t=0$. The disc evolves under the influence of pressure, viscosity, and the combined gravitational force of the central star and companion object. The orbit of the star-companion system evolves due to the gravity of the disc. We considered companion masses in the range $1 \leq m_{p} \leq 30$ Mupiter.

For $m_{p}=1 \mathrm{M}_{\text {Jupiter }}$, we find that the orbit remains essentially circular. This calculation is described in Nelson et al. (2000). The results of the remaining calculations are shown in figure 1. A transition in behaviour occurs for $m_{p} \gtrsim 20$ $\mathrm{M}_{\text {Jupiter }}$, such that rapid eccentricity growth occurs for masses above this value. We estimate that the eccentricity growth saturates at $e \lesssim 0.25$. A time sequence showing the evolution of the disc for the case $m_{p}=30 \mathrm{M}_{\text {Jupiter }}$ is shown in figure 2. A complete description of this work is presented in Papaloizou, Nelson, \& Masset (2000).

In addition to there being a growth of orbital eccentricity for $m_{p} \gtrsim 20$ $\mathrm{M}_{\text {Jupiter }}$, we find a growth in disc eccentricity, such that the inner parts of the outer disc become eccentric (Papaloizou et al. 2000). The combined growth of orbital and disc eccentricity leads to a break down in the tidal truncation of 


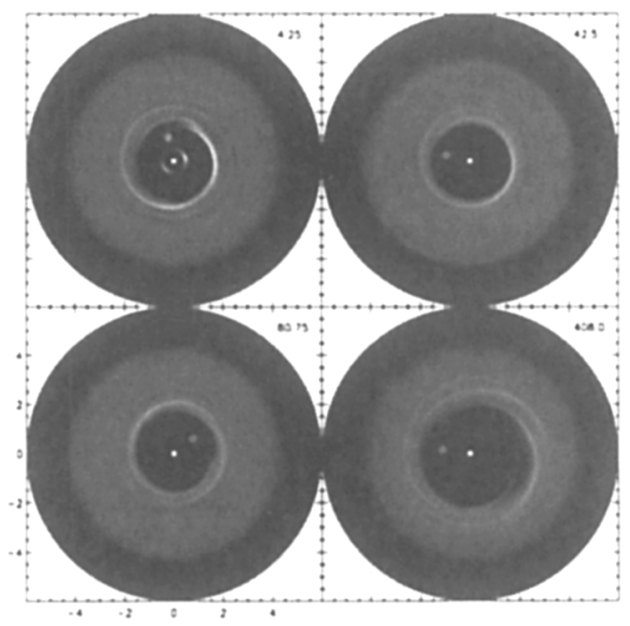

Figure 2. This figure shows the evolution of the disc surface density profile for the case $m_{p}=30 \mathrm{M}_{\text {Jupiter }}$. The initial clearing of a gap is shown in the first and second panels. The growth of disc eccentricity interior to the 1:3 resonance (located at $r \simeq 2.08$ ) may be observed in the third and fourth panels. Times are shown in units of the orbital period.

the disc, such that the companion object will be able to accrete efficiently from the disc. We suggest that this may allow companions of brown dwarf mass to grow towards being of stellar mass, and may therefore explain the 'brown dwarf desert'.

\section{References}

Artymowicz, P. 1992, PASP, 104, 759

Bryden, G., Chen, X., Lin, D.N.C., Nelson, R.P., \& Papaloizou, J.C.B. 1999, ApJ, 514, 344

Kley, W. 1999, MNRAS, 303, 696

Lin, D.N.C., \& Papaloizou, J.C.B. 1986, ApJ, 309, 846

Lubow, S.H., Seibert, M., \& Artymowicz, P. 1999, ApJ, 526, 1001

Marcy, G.W., Cochran, W.D., \& Mayor, M. 2000, Protostars \& Planet IV, (Tucson: University of Arizona Press; eds. Mannings, V, Boss, A.P., Russel, S.S), p.1285

Nelson, R.P., Papaloizou, J.C.B., Masset, F., \& Kley, W. 2000, MNRAS, 318, 18

Ziegler, U., \& Yorke, H.W. 1997, Comp. Phys. Comm., 101, 54 